\title{
Role of MRI in Diagnostic Evaluation of Different Disorders
}

Batchu Pavani ${ }^{\star}$, Ramesh G, Satheesh Gottipati and Srinivasa Babu P

Vignan Pharmacy College, Vadlamudi, Andhra Pradesh, India

*Corresponding author: Batchu Pavani, Vignan Pharmacy College, Vadlamudi-522213, Andhra Pradesh, India, Tel: +919014763255; E-mail: bpavani97@gmail.com Rec Date: October 26, 2018, Acc Date: December 06, 2018, Pub Date: December 10, 2018

Copyright: (C) 2018 Pavani B, et al. This is an open-access article distributed under the terms of the Creative Commons Attribution License, which permits unrestricted use, distribution, and reproduction in any medium, provided the original author and source are credited.

\begin{abstract}
MRI (Magnetic Resonance Imaging) is a radiology technique. MRI is based on NMR principles .In 1997 the first MRI exam was performed on a human being. People who are suffering from sport injury, musculoskeletal problems, spinal injuries, prostate problems can be diagnosed by MRI.
\end{abstract}

Keywords: MRI; Musculoskeletal problems; Spinal injuries

\section{Introduction}

MRI is a non-invasive imaging technique capable of detailed tissue characterization and blood flow measurements. The procedure involves placing patient in a device generating a powerful magnetic field and aligning the protons of the body's hydrogen atoms relative to the magnetic field [1]. Radio waves pulsed through the field force the proton to shift their orientation when the radio waves stop, the protons return to their previous orientation releasing energy in the form of radio waves. The waves are detected by a scanner and converted into images. The images are physiologically gated to on ECG.

The equipment involves a hallow tube which is surrounded by a large cylindrical magnet. In this procedure, the patients should remain motionless. MRI equipment provides a magnetic field around the body strong enough to temporarily realign water molecule in the tissues. Radio waves are then passed through the body to detect the relaxation of the molecule back to random alignment and trigger a resonance signal at different angles with in the body [2]. A contrast dye may be used to enhance visibility of certain areas or tissues. Sedation may be necessary. In addition, patients with mental prosther (Pacemakers and ferrous magnetic intracerebral clips) should not undergo.

Clinical uses of cardiac MRI include assessment of congenital, aortic and pericardial diseases, tumour and IV thrombus. MRIs has become more available, but restricted to some medical center because of the cost, scan time, and need for specialized equipment and personal. Current recommendations for CMR use include the evaluation of chest pain syndrome with use of vasodilator perfusion. CMR or Dobutamine stress function CMR in patients with intermediate pretest probability of ischemic heart disease in the setting of non-interpretable electrocardiogram or inability to exercise or for the evaluation of suspected coronary anomalies. There is no clear acceptable use of CMR in the settings of acute chest pain.

\section{Literature Review}

\section{Role of MRI in diagnostic evaluation of different disorders}

Role of MRI in the evaluation of painful hip joint (MRI of hip joint): This ball and socket joint [3] is formed by the cup-shaped acetabulum of the innominate (hip) bone and the almost spherical head of the femur. The capsular ligament encloses the head and most of the neck of the femur. The cavity is depended by the acetabular labrum, a ring of fibro-cartilage attached to the rim of the acetabulum, which stabilizes the joint without limiting its range of movement.

MRI is very useful in characterizing various disorder and helpful in assessing the extent of chondral, soft tissue involvement and osseous. MRI studies can accurately demonstrate articular cartilage abnormalities, synovial proliferation, ligaments, joint effusions, and juxta-articular soft tissue [4]. MRI is the best method of choice in evaluating the various disorder of hip joint and provides accurate imaging in assessing the extent of chondral, soft tissue and osseous MRI of hip joint is informative and provides accurate for assessing of hip pain.

Role of MRI in the evaluation of muscle disease: Muscle disease occurs in all age groups and can cause serious physical disability. Some muscle disease responds well to medical treatment, while many of the physical disabilities can be improved or prevented. There are number of muscle disease which includes: Myopathy, Muscular dystrophy, Myasthenia gravis, muscle weakness [5] etc. MRI of muscle is a useful tool in diagnosing the patients with muscle dystrophies. MRI of muscle provides many structural aspects and function of skeletal muscle.

Role of MRI in evaluation of spinal tumours: Spinal tumours are neoplasms which are located in the spinal cord. Depending on their location, spinal tumours can be Extradural (Outside the dura mater lining), Intradural (part of dura), Intramedullary (inside the spinal cord), Extramedullary (inside the dura but outside the spinal cord). The most common type of tumour is Extradural tumour. MRI in spinal tumour: The main aim of the study is to assess and evaluate the spinal tumour by MRI. Spinal tumours can significantly cause morbidity and associated with mortality as well. MRI of spinal tumour is very helpful to classify the tumour into different compartments [6,7] (extradural, intradural extramedullary and intradural intramedullary compartments).

Role of MRI in evaluation of seizures: Seizure is a sudden uncontrolled electrical disturbance in the brain. It can cause changes in behaviour, movements or feelings and in level of consciousness. If two or more seizures or tendency to have seizure then it is called epilepsy [8]. Seizure can happen after a stroke, closed head injury, an infection such as meningitis or other illness. The cause of seizure is unknown. MRI plays a crucial role in evaluating the patients with seizures. It has been showed that MR is positive in myoclonic seizures, absence 
seizures and temporal lobe seizures. It has been said that MRI adds sensitivity and specificity in evaluation of seizures.

Role of MRI in evaluation of Alzheimer's disease: A progressive disease that destroys memory and other important mental functions. There is no cure for Alzheimer's disease, but treatments for symptoms are available and research continues. Although current Alzheimer's disease treatment cannot stop Alzheimer's from progressing, they can temporarily [9] slow the worsening of dementia symptoms and improve quality of life for those with Alzheimer's and their care takers. The main aim of the study is to identify people with mild MCI (mild cognitive impairment) due to Alzheimer's disease.

Role of MRI in evaluation of psychiatric disorder: Schizophrenia is a mental disorder characterised by abnormal social behaviour and failure to understand reality. People with schizophrenia often have mental health problems such as anxiety, depression, or substance abuse. Symptoms typically come on gradually, begin in young adulthood and last a long time. The cause of schizophrenia includes genetic and environmental factors. MRI in schizophrenia has provided valuable insight to the degenerative processes of brain and has allowed for better understanding of the speed with which brain tissue loss occurs, allowing for study and potential [10] development of novel pharmaco-therapeutics which may help deter the advancement of brain volume loss in these patients.

Advancement in MRI and PET technologies along with simultaneous development of novel imaging sequences or contrasting agents have increases resolution and sensitivity of imaging for amyloid beta peptide. This is expected to improve the imaging diagnosis scenario. Various molecular markers have been regulated and catered for to predict the incident cases and in therapeutic trials.

New research in MRI have decreased the utility of structural imaging as clinicians require a more convenient and reliable method to supplement structural imaging method to accurately estimate hippocampal size. Recent developments may help in reaching but confirmatory trials needs to be performed before commercialization. Image quantification is done clinically and demands considerable processing time and operator attention.

\section{Discussion and Conclusion}

MR studies have been started performing since 30 years and have played an important role in removing the societal stigmas by helping to record structural and functional differences in the patients. The discovery of various MR equipment and MR techniques along with the diversity of research methods used is a pro and con. As it allows flexibility yet do not allow the translation of MR findings in individual patients. Howsoever the present condition MR finding patterns have the potential to play the role of biomarkers in various diseases.

A multifaceted approach in MR research will bring new perspectives and help in development of evolving medical knowledge. MR research outcomes may look glazing at first but on analysis shows various abnormalities due to various software glitches. These emerging patterns of abnormalities must be studies and the bias must be removed to get absolute results. New advances in MR technology is helping further in removing this bias. New technology involves higher magnetic field strength units that decreases signal-to-noise ratio and improves visualization of anatomic, functional, and/or spectral resolution. However, care should be taken to ensure that these new technologies do not introduce additional variability that undermines their advantages. Experiments must be done to find out advanced statistical and data-processing techniques that improves survey and research data visualization. Techniques like principles components analysis is supposed to help in establishing MR-based signatures of various diseases to facilitate its diagnosis in individual patients.

\section{References}

1. Lee M (2009) Basic skills in interpreting laboratory data. ASHP.

2. Hachinski V, Iadecola C, Petersen RC, Breteler MM, Nyenhuis DL, et al. (2006) National Institute of Neurological Disorders and Stroke-Canadian Stroke Network Vascular Cognitive Impairment Harmonization Standards. Stroke 37: 2220-2241.

3. Waugh A, Grant A (2006) Ross and Wilson: Anatomy and Physiology in Health and Illness. (10th edn) Churchill Livingstone, USA. 2: 76-129.

4. http://www.sciencepublishinggroup.com/journal/index?journalid=156.

5. https://www.nni.com.sg/patientsandvisitors/conditions-treatments/pages/ muscle-diseases.aspx

6. https://www.mayoclinic.org/diseases-conditions/spinal-cord-tumor/ symptoms-causes/syc-20350103.

7. Ravi N, Manjappa BH, Nagaraj BR, Naveen KG, Lakshmeesha MT (2014) MRI evaluation of different spectrum of spinal tumors. SSRG International Journal of Medical Science 1: 12-23.

8. https://www.mayoclinic.org/diseases-conditions/seizure/symptomscauses/syc-20365711.

9. https://www.mayoclinic.org/diseases-conditions/alzheimers-disease/ symptoms-causes/syc-20350447.

10. Crossley NA, Scott J, Ellison-Wright I, Mechelli A (2015) Neuroimaging distinction between neurological and psychiatric disorders. Br J Psych 207: 429-434. 